\title{
Evaluating the Influence of Defects on the Young's Modulus of Carbon Nanotubes Using Stochastic Modeling
}

\author{
Roham Rafiee*, Reza Pourazizi \\ Composites Research Laboratory, Faculty of New Sciences \& Technologies, \\ University of Tehran, 1439955941, Tehran, Iran
}

Received: October 25, 2013; Revised: April 9, 2014

\begin{abstract}
The main goal of this research is to investigate the influence of structural defects on the mechanical properties of single-walled carbon nanotubes (CNTs). Two different types of the structural defects called Stone-Wales and vacancy defect are studied. While the former is categorized under the processinduced defect and it appears during the growth process of CNT, the later is caused when chemical functionalization is applied to $\mathrm{CNT}$ for fabrication carbon nanotube reinforced nanocomposite providing better bonding between CNT and surrounding resin. The number of broken C-C bonds, distributions and their orientations are all taken into account as random variables accounting for full stochastic analysis. Therefore, a computer code is provided for the stochastic modeling. The finite element (FE) model of the CNT is built using nanoscale continuum mechanics approach and then structural defects are applied randomly to the CNT. The Young's modulus of defected CNTs are obtained and compared with non-defected ones. It is revealed that the importance of vacancy defect is considerably higher than that of Stone-Wales defects implying on the drawback of chemical functionalization process. A detailed study is carried out on the topology of the defect and also continuous probability density functions of defect CNT Young's modulus are characterized.
\end{abstract}

Keywords: carbon nanotube, defect, finite element modeling, young's modulus, stochastic analysis

\section{Introduction}

Nowadays, supreme and exceptional properties of carbon nanotubes (CNTs) from different mechanical, thermal and electrical viewpoints have encouraged researchers to incorporate them into polymeric resin generating new category of advanced materials as nanocomposites ${ }^{1,2}$. Experimental studies have been revealed that by adding small portions of CNTs into polymeric resin mechanical properties of the nanocomposites have been enhanced considerably ${ }^{3,4}$. The efficiency of CNTs in reinforcing polymer depends on the atomic structure of CNTs at nano-scale, while load transferring phenomenon from matrix to CNT through the intermediate phase between CNT and surrounding polymer plays a key role at the scale of micro in this regard.

Containing $\mathrm{SP}^{2}$ bonds, the atomic structure of CNTs cannot establish strong covalent bond with surrounding polymer. Consequently, the load transferring mechanism from matrix to CNT is naturally limited to weak nonbonded van der Waals (vdW) interaction ${ }^{5,6}$. Thanks to chemical functionalization, some researchers tried to improve load transferring mechanism by providing cross covalent links between the carbon atoms of CNT and the polymer chain of the matrix ${ }^{5-8}$. In this process, some $\mathrm{C}-\mathrm{C}$ bonds on CNT nano-structure are broken and associated carbon atoms with broken bonds will be connected to the polymer chains through covalent cross bonding. This will be led to enhancement in load/stress transfer from the resin to CNT resulting in improved mechanical properties of nanocomposites ${ }^{7,8}$. The functionalization procedure has a

*e-mail: Roham.Rafiee@ut.ac.ir main shortcoming by causing defects in the nano-structure of CNT which may significantly reduce Young's modulus of CNT. Moreover, almost all produced CNTs accommodate some processing-induced defects occurring during their growth process. Therefore, it is of great importance to study the degree to which these structural defects will affect mechanical properties of CNT. Hao et al. ${ }^{9}$ investigated the influence of defects on the mechanical properties of $(12,12)$ and $(7,7)$ CNTs with molecular dynamic (MD) method and reported reduction in Young's modulus about 5\% and 4\% for $(12,12)$ and $(7,7) \mathrm{CNTs}$, respectively, by adding one vacancy defect on their structures. Yuan and Liew ${ }^{10}$ did the same work and used MD to investigate the Young's modulus of $(5,5)$ and $(10,10)$ CNTs with some vacancy defects and reported the reduction of the Young's modulus too. Ghavamian and his coworkers ${ }^{11}$ by finite element method (FEM) achieve the same result for reduction of Young's modulus due to existence of defects. All of this studies considered the vacancy defect type in CNT nano-structure and use deterministic analysis. Limited researches have been devoted to the influence of CNT defects on other properties like thermal conductivity ${ }^{12}$.

It is worth noting that other parameters like nonstraight shapes of CNTs due to their high aspect ratios, agglomeration of CNTs and non-uniform dispersion of CNTs in resin are also important parameters defining the efficiency of CNT in reinforcing resin at meso and macro scales, respectively ${ }^{13,14}$.

In this article, the influence of structural defects on the longitudinal Young's modulus of CNT is investigated using 
nano-scale continuum mechanics approach with aid of finite element modeling. Firstly, a prefect CNT is simulated and then structural defects are applied to the model randomly. Then, obtained results are analyzed and discussed. Finally, due to conducting stochastic analysis, the results are examined statistically.

\section{CNT Morphology and Defect Types}

CNTs can be virtually assumed as a rolled graphene sheet into a hollow cylinder. In this structure, each carbon provides three covalent bonds to the neighboring carbon atoms. Atomic structure of CNT is described by chirality vector as it follows ${ }^{2}$ :

$$
\vec{C}_{h}=n \vec{a}_{1}+m \vec{a}_{2}
$$

In which $n$ and $m$ are integer numbers and $a_{1}$ and $a_{2}$ are The unit vectors shown in Figure 1.

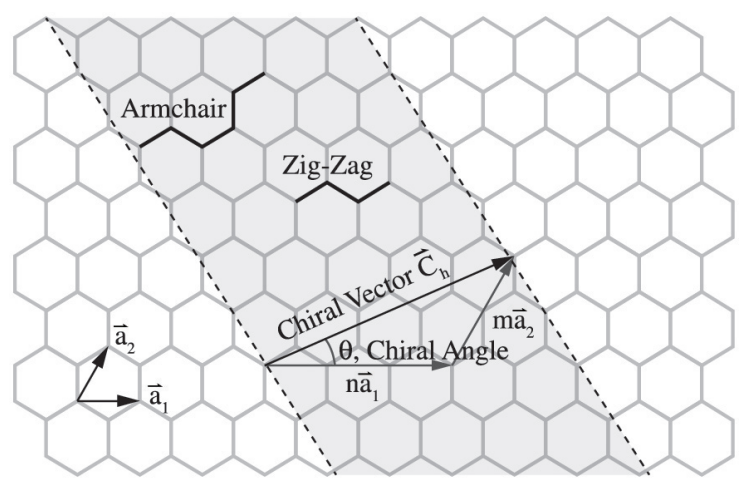

Figure 1. Schematic representation of rolling graphene layer to create $\mathrm{CNT}^{15}$.

In Figure 1, $\theta$ is chiral angle and varies between 0 and 30 degree. Three different types of CNTs introduced as zigzag, armchair and chiral are characterized by zero, 30 and a value between zero and 30 degrees, respectively, for the chiral angle. Chiral index in the form of $(n, 0)$ denoted to zigzag while $(n, n)$ is representative for the armchair. The chiral index of $(n, m)$ whrerin descrive chiral CNT. Figure 2 demonstrates three types of single walled CNT.

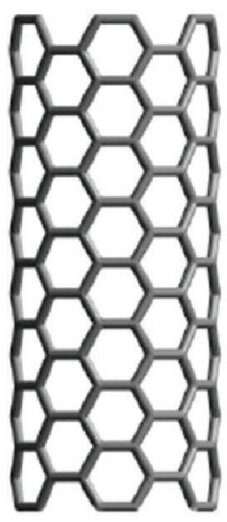

Armchair

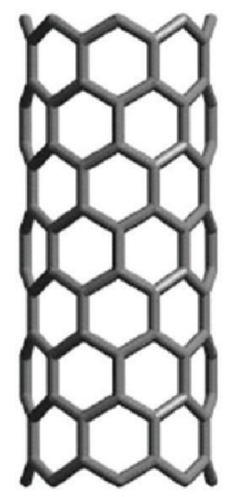

Zigzag

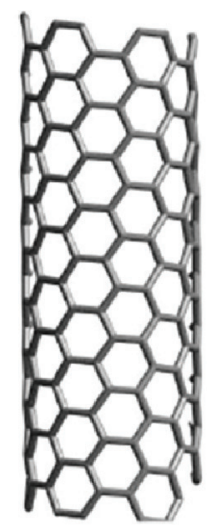

Chiral
The structural defects of CNT can be divided in two main categories. The first category known as Stone-Wales (S-W) structural defects are intrinsic defects arisen from growth process of CNT. In S-W defects two C-C bonds are broken and two other bonds are formed in a manner that four hexagons in CNT structure are converted to two Heptagons and two Pentagons. Thus, this defect is called 5-7-7-5 defect as depicted in Figure 3a. Another type of S-W defect is created when $\mathrm{C}_{2}$ dimer removed from a pentagon structure of CNT cap and new bonds are formed. This defect is known as 5-8-5 structural defect which is shown in Figure $3 b$. The 5-8-5 defect appears in the cap of CNT.

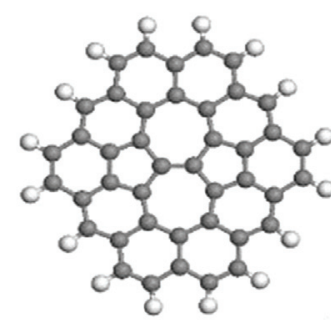

(a) $5-7-7-5$

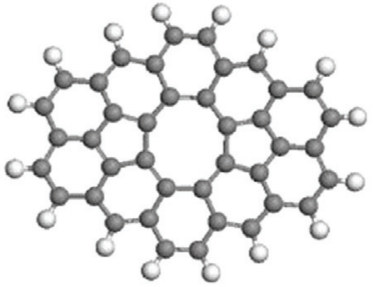

(b) $5-8-5$
Figure 3. Stone-Wales defect in CNT structure ${ }^{17}$.

The second category of CNT defect known as Vacancy defect is provided during the chemical functionalization of CNT as illustrated in Figure 4. This defect can be also appeared during the processing of multi-walled CNT.

\section{Modeling Non-defected CNT}

Due to the associated challenges with the experimental study at the scale of nano, theoretical modeling techniques play an important role in understanding the mechanical behavior of nanostructures like CNTs. Generally, simulation of CNT at nano-scale is either conducted using atomistic modeling or continuum modeling. In atomistic modeling, the Schrödinger equation is solved directly or is replaced with Newton' second law to extract the positions of atoms. Huge amount of calculation, limitation to very small length and short time scales and also complicated formulation stimulated researchers to employ continuum modeling in the both forms of analytical and numerical for this purpose ${ }^{18}$. In this research, the nanoscale continuum modeling is used. In this method, instead of replacing the structure of CNT with a continuum cylinder structure (solid or hollow), The C-C bonds of CNT atomic structure is replaced by a continuum beam element. The advantage of this method lies in the fact that the lattice structure of CNTs are kept in the model. The nano-scale continuum mechanics approach has been originally developed by $\mathrm{Li}$ and $\mathrm{Chou}^{19}$ and successfully applied to CNT. The mechanical properties of beam elements representing $\mathrm{C}-\mathrm{C}$ bonds are obtained by a correlation between interatomic potential energy in molecular space and strain energy of beam element in structural mechanics space ${ }^{20}$. Mechanical and geometrical properties of the elements are calculated by using the relationship developed by $\mathrm{Li}$ and $\mathrm{Chou}^{19}$ as listed in Table 1 on the basis of linear interatomic potentials.

Figure 2. Different types of $\mathrm{CNT}^{16}$. 

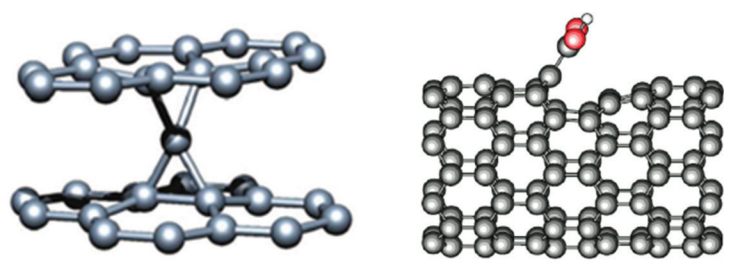

Figure 4. The vacancy defect in CNT structure.

Table 1. Mechanical and geometrical properties of elements.

$\begin{array}{cc}0.147[\mathrm{~nm}] & \text { Element diameter } \\ 5.48[\mathrm{TPa}] & \text { Elastic modulus } \\ 0.871[\mathrm{TPa}] & \text { Shear modulus } \\ 2.15 & \text { Poisson's ratio }\end{array}$

As previously mentioned, carbon nanotubes can be virtually imagined as a rolled graphene sheet. Thus, the coordinate of CNT atoms can be obtained from the coordinate of carbon atoms in a graphene sheet using below mapping equation ${ }^{20}$ :

$$
(X, Y, Z)=\left[R \cos \left(\frac{x}{R}\right), R \sin \left(\frac{x}{R}\right), y\right]
$$

The coordinate of carbon atoms obtained from Equation 2 which are describing the location of nodes of the finite element model are entered into the ANSYS commercial FE package ${ }^{21}$. Each node is connected to other three adjacent nodes using built-in BEAM4 element of ANSYS via developed macro. This BEAM4 element has six degree of freedom at each node. The length of CNT is taken 10 times greater than CNT diameter to avoid the numerical errors caused by the effects of boundary conditions on the overall structural behavior.

Finite element models of the zigzag and armchair CNTs with different diameters are made to extract the longitudinal modulus of CNTs. For this purpose, one end of CNT is completely restrained from any movement while uniform axial displacement is applied to the other end of the model. The elastic modulus of investigated CNT can be obtained using Equation 3:

$$
E_{C N T}=\frac{\sigma_{Z}}{\varepsilon_{Z}}=\frac{\frac{\sum f_{Z}}{A}}{\Delta L / L}
$$

where $f_{\mathrm{z}}$ represents the reaction force at applied constraints to the model and obtained from the output of the finite analysis and $A$ stands for the cross section of CNT calculated by Equation 4:

$$
A=\pi \times\left[(R+t / 2)^{2}-(R-t / 2)^{2}\right]
$$

where $t$ and $R$ denotes the thickness and radius of the CNT respectively. There is not any consistent value for the wall thickness of CNT in literature. Here, the widely accepted value of $0.34 \mathrm{~nm}$ is chosen as interplaner spacing between adjacent graphene sheets in graphite.

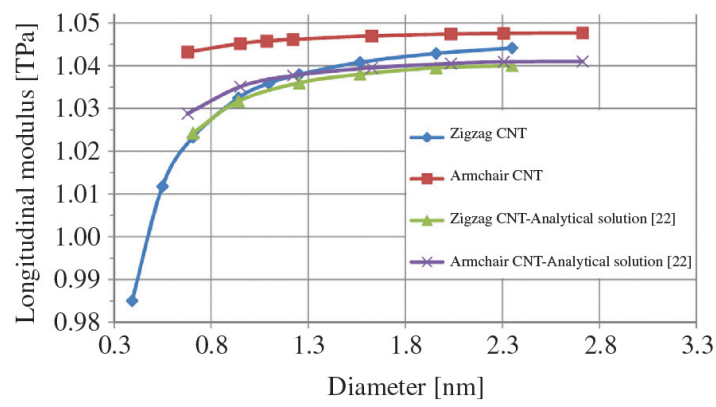

Figure 5. The longitudinal modulus of CNT in term of diameter.

The obtained results for the Young's modulus of nondefected CNTs in comparison with available analytical solution in literature are presented in Figure 5. It is worth mentioning that the analytical solution presented in Figure 5 for the sake of comparison was also developed for a discrete structure of CNT on the basis of nanoscale continuum approach using beam structural member ${ }^{22}$.

Good agreement between obtained results by FE analysis and reported results by analytical solution implies on the sufficient level of accuracy for employed modeling procedure.

\section{Investigation of Vacancy Defect}

In order to investigate the effect of vacancy structural defects on the elastic modulus of CNT, a Macro was written in ANSYS. This computer code randomly removes C-C bonds in the CNT nanostructure on the basis of MonteCarlo technique ${ }^{23}$. Two parameters of the distribution and the position of removed $\mathrm{C}-\mathrm{C}$ bonds along the axial and circumferential directions of the CNT are selected randomly. Then same modeling and computational procedure which is already explained in preceding section is followed here to obtain the Young's modulus of defected CNT. Due to the stochastic implementation of modeling procedure arisen from two random variables, the code is repeated sufficiently for defined number of defects; until the standard deviation (SD) of obtained results is less than $1 \%$ as convergence criterion. Finally, the average of Young's modulus obtained from different realizations is reported as the Young's modulus of the investigated defected CNT. It should be noted that if the removed element is placed at very both ends of the model, it will influence on the results because of the end effect. Thus, the model is checked carefully prohibiting such a specific located defect. The flowchart of the stochastic code simulating CNT with vacancy defect is mentioned in Figure 6.

The defect density is defined to express the amount of incorporated defects as bellow:

$D=\frac{N_{d}}{N_{t}}$

where; $N_{\mathrm{d}}$ and $N_{\mathrm{t}}$ are the number of removed bonds and the total bonds of CNT before incorporating defects, respectively. 


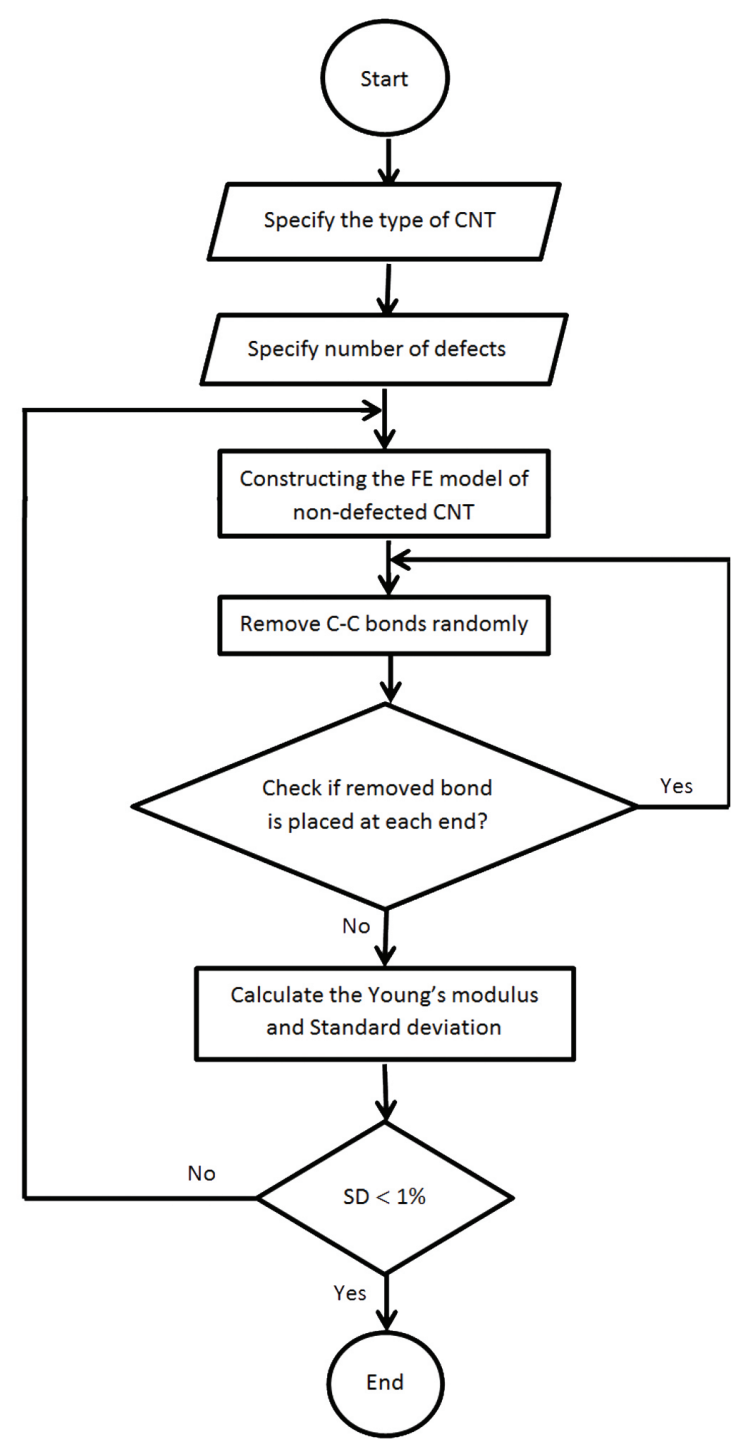

Figure 6. Flowchart of modeling and analysis process of defected CNT.
The convergence trend for a case study on a CNT with $(12,0)$ chiral index containing and $4 \%$ defect density is demonstrated in Figure 7.

As is clear from Figure 7, the defined convergence criterion is met after executing the code 450 times. The written code is executed to evaluate the effect of vacancy defects on different CNTS with chiral indices of $(0,9),(0$, $12),(5,5),(7,7)$ and $(9,9)$. The obtained results versus the defect density is presented in Figure 8.

It can be understood from Figure 8 that the longitudinal elastic modulus of almost all CNTs decreases about $6 \%$ and $12 \%$ by removing $1 \%$ and $2 \%$ of $\mathrm{C}-\mathrm{C}$ bonds, respectively. A monotonically linear decreasing trend can be considered with a good level of accuracy for reduction in CNT Young's modulus with respect to the defect density.

Yuan and Liew ${ }^{10}$ used molecular dynamics to investigate the reduction in Young's modulus of $(5,5)$ and $(10,10)$ CNTs with some vacancy defects. They applied 1 to 8 percent of vacancy defects on CNT and reported the reduction of the Young's modulus.

Ghavamian and his coworkers ${ }^{11}$ studied the vacancy defects in CNT using FEM. They considered the vacancy defect as vacancy of carbon atoms in CNT structure and investigated only the vacancy defects caused by CNT processing by removing nodes of the model randomly which has been led to removal of three neighboring C-C bonds and the defects caused by functionalization have not been studied.

In their study, the Young's modulus of the zigzag CNT with $1 \%$ vacancy defects decreased about $10 \%$ and for Armchair CNT with this amount of defects the Young's modulus decreased about $9 \%$.

Comparison between results presented in published data and the results obtained in this study is presented in Table 2. Some researchers have only studied one CNT type, but in current research different CNTs are studied. Therefore; the reported reduction of modulus in this study is presented as a ragne of reduction. The obtained results in this study are in good agreement with what reported using molecular dynamics and finite element methods.

Table 2. Results comparison for CNT with vacancy defect.

\begin{tabular}{|c|c|c|c|c|c|}
\hline $\begin{array}{l}\text { Reduction of modulus } \\
\text { in this study [\%] }\end{array}$ & $\begin{array}{l}\text { Reduction of } \\
\text { modulus [\%] }\end{array}$ & Method & $\begin{array}{l}\text { Number/Percent } \\
\text { of vacancy defect }\end{array}$ & CNT type & Researcher (s) \\
\hline $0.27-0.67$ & 0.9 & MD & 1 & Armchair & Saxena and $\mathrm{Lal}^{24}$ \\
\hline $0.6-2$ & 2.9 & MD & 3 & Armchair & Sharma et al. ${ }^{25}$ \\
\hline $0.6-2$ & 3 & \multirow{2}{*}{ MD } & 3 & \multirow{2}{*}{ Armchair } & \multirow{2}{*}{ Chen et al. ${ }^{26}$} \\
\hline $1-4.9$ & 4.8 & & 6 & & \\
\hline 7.1 & 7.9 & \multirow{2}{*}{ MD } & $1 \%$ & \multirow{2}{*}{ Armchair $(5,5)$} & \multirow{2}{*}{ Yuan and Liew ${ }^{10}$} \\
\hline 12.5 & 15.8 & & $2 \%$ & & \\
\hline 6 & 8.7 & \multirow{2}{*}{ FEM } & $1 \%$ & Armchair $(10,10)$ & \multirow{2}{*}{ Ghavamian et al. ${ }^{11}$} \\
\hline 6.1 & 9.4 & & $1 \%$ & Zigzag $(14,0)$ & \\
\hline
\end{tabular}




\section{Investigation of Stone-Wales Defect}

The influence of S-W defect in the form of 5-7-7-5 on $(20,20)$ and $(12,0)$ CNTs is studied. For this purpose, after modeling non-defected CNTs, the S-W defect is applied to model randomly. The same convergence criterion defined in previous section, i.e. standard deviation of longitudinal modulus less than $1 \%$, is also utilized in this section. The code was executed 20 times to meet the mentioned convergence criterion. For instance, two finite element models of defected CNT containing S-W defect is shown in Figure 9.

The results of this analysis are presented in Table 3 .

The obtained results in this research are compared with reported results by Xiao and Gillespie ${ }^{27}$ and presented in Table 4. They used finite element method to study the effect of S-W defects ${ }^{27}$.

According to the presented results in Table 3, it is observed that the level of reduction in Young's modulus

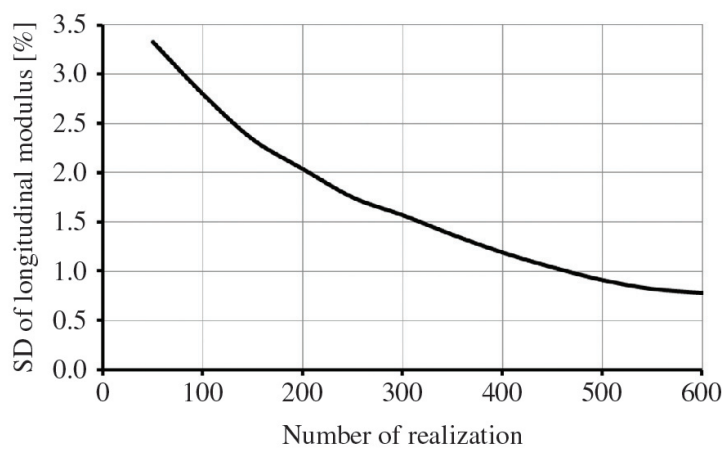

Figure 7. The convergence results in terms of number of runs.

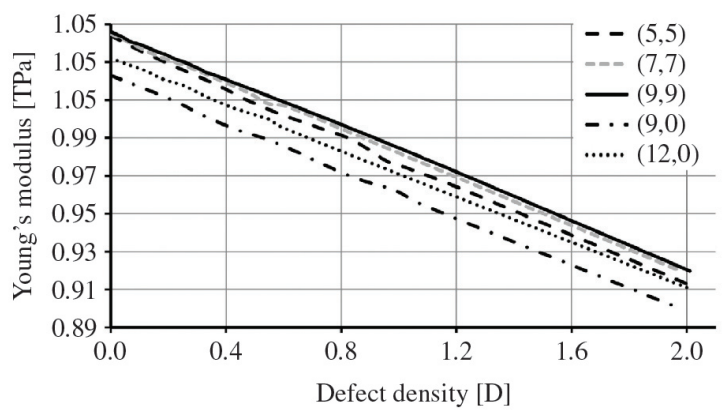

Figure 8. The Young's modulus of defected CNTs.
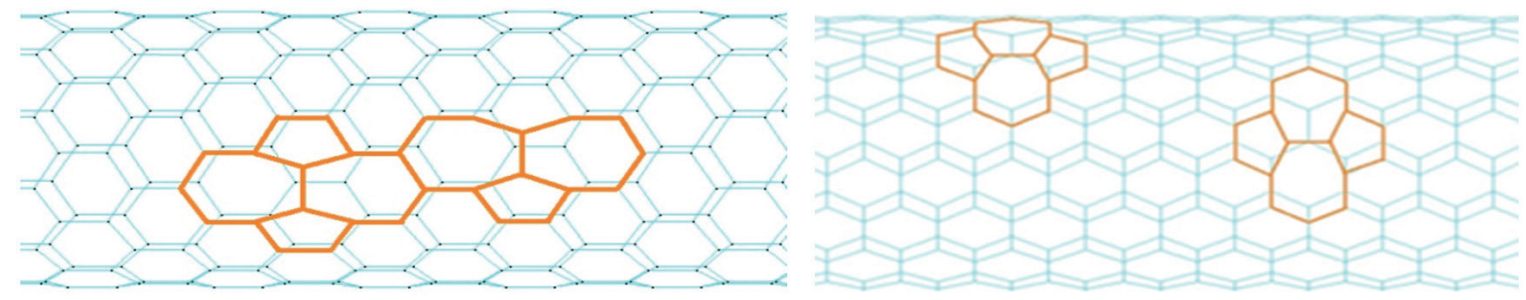

of Zigzag nanotubes is more than that of Armchair ones. It is also found out that the rate of reduction in Young's modulus of CNT will decrease by increasing the number of S-W defects. The reduction rate of Young's modulus for $(20,20) \mathrm{CNT}$ in term of the number of S-W defects is drawn in Figure 10.

Comparing presented results in Tables 2 and 3, it is clear that the influence of the vacancy defect on the reduction of CNT Young's modulus is much more than that of S-W defects. So it could be inferred that chemical functionalization of CNT will decrease the longitudinal modulus of a CNT much more severely than inherent defects caused during CNT growth process. This was also reported in literature $\mathrm{e}^{25,27}$.

\section{Topology of Vacancy Defect}

In the previous section, the global influence of vacancy defect on the reduction of CNT Young's modulus was studied. In this section, a detailed study is conducted on the location and arrangement of removed bonds in CNT addressing the minimum and the maximum reduction in modulus. A discussion will be carried out on $(9,0)$ and $(5$,

5) CNTs as case studies.

Breaking one $\mathrm{C}-\mathrm{C}$ bond in a $(9,0) \mathrm{CNT}$, the highest and the lowest elastic modulus is achieved as 1.019 and 1.011 $\mathrm{TPa}$, respectively. The corresponding defected CNTs with aforementioned results are shown in Figure 11. It can be understood that the lowest modulus of CNT is achieved when the axial C-C bond is broken.

This can be justified by this fact that in zigzag CNTs, the axial bonds contributes to load carrying capacity much more than inclined bond with respect to the axial axis. Another evidence for this issue can be also observed for the case of removing three bonds which is depicted in Figure 12.

Table 3. Young's modulus of CNTs containing Stone-Wales defects.

\begin{tabular}{cccc}
\hline $\begin{array}{c}\text { Reduction in } \\
\text { modulus [\%] }\end{array}$ & $\begin{array}{c}\text { Average of Young's } \\
\text { modulus [GPa] }\end{array}$ & $\begin{array}{c}\text { Number of } \\
\text { S-W defects }\end{array}$ & CNT \\
\hline 0.26 & $1029.8 \pm 1.2$ & 1 & \\
0.46 & $1027.8 \pm 1.3$ & 2 & $(12,0)$ \\
0.59 & $1026.4 \pm 1.5$ & 4 & \\
\hline 0.2 & $1045.6 \pm 0.8$ & 1 & \\
0.31 & $1044.4 \pm 1.1$ & 2 & $(20,20)$ \\
0.44 & $1043.1 \pm 1.3$ & 4 & \\
0.63 & $1041.1 \pm 2.2$ & 6 & \\
\hline
\end{tabular}

Figure 9. Finite element models of CNT incorporating S-W defects $(20,20)$ (right) and $(12,0)$ (left). 
For the case of Armchair CNT, it is observed that inclined covalent bonds play more important role than perpendicular bonds to the axial axis in load carrying capacity of the nanotube. The topology of defects leading to the maximum and minimum reduction is mentioned in Figure 13 when four covalent bonds are removed.

In addition to the orientations of the covalent bonds, it is also observed that the level of reduction in CNT Young's modulus is sensible to the distribution of the broken bond along the CNT length. Namely, if all broken bonds are placed at the same length all around the CNT circumference, the minimum Young's modulus will be obtained implying on the maximum reduction, however, if all broken bonds are distributed along the whole length of CNT instead of

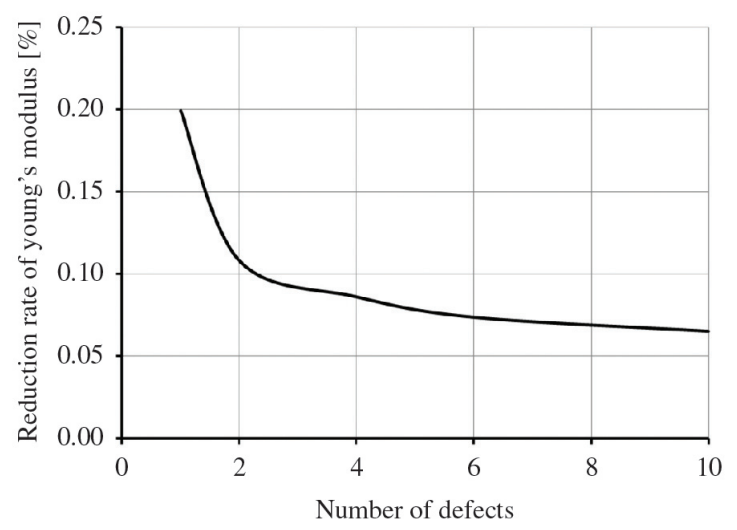

Figure 10. Trend of Young's modulus reduction for $(20,20)$ CNT with S-W defects. concentrating at the local position, this will be led to the maximum Young's modulus as a minimum reduction level. In general, concentration of the broken bonds in local region will considerably reduce the Young's modulus of defected CNT in comparison with non-defected one. This behavior was also reported in literature using molecular dynamics simulation $^{28}$.

\section{Probabilistic Analysis of Results}

As it is explain in the flowchart of the modeling presented in Figure 6, stochastic modeling is conducted considering number, orientations and positions of defects as random variables. Therefore, several realizations are required to meet the mentioned convergence criterion. Statistical analysis of obtained results will be discussed in this section. The case study is performed on a defected CNT with $(12,0)$ chiral index and with $5 \%$ defect density. For this CNT, the code is generated 600 samples to achieve standard deviation of Young's modulus less than $1 \%$. Among these results, the minimum modulus is $602.7 \mathrm{GPa}$ while the maximum modulus is reported as $800.5 \mathrm{GPa}$. The probability distribution of results is presented in Figure 14.

The Pareto plot of presented results in Figure 14 is shown in Figure 15. In this plot, the frequencies of elastic moduli are sorted in ascending order and the accumulation percent of these modulus are shown too.

According to statistical data and aforementioned diagrams, the mode value of $0.75 \mathrm{TPa}$ and the mean value of $0.74 \mathrm{TPa}$ are achieved for the Young's modulus of investigated defected CNT. Since, mode value and mean

Table 4. Comparing the results of the Stone - Wales defect modeling.

\begin{tabular}{|c|c|c|c|c|}
\hline \multicolumn{2}{|c|}{ Reduction in Young's modulus [\%] } & \multirow[b]{2}{*}{$\begin{array}{l}\text { Number of } \\
\text { defects }\end{array}$} & \multirow[b]{2}{*}{$\begin{array}{l}\text { Young's modulus (non- } \\
\text { defected CNT) [TPa] }\end{array}$} & \multirow[b]{2}{*}{$\begin{array}{l}\text { Type of } \\
\text { CNT }\end{array}$} \\
\hline Present study & $\begin{array}{c}\text { Xiao and Gillespie's } \\
\text { research }^{26}\end{array}$ & & & \\
\hline 0.4 & 0.35 & 4 & \multirow{2}{*}{1.13} & \multirow{2}{*}{$(20,20)$} \\
\hline 0.5 & 0.6 & 6 & & \\
\hline
\end{tabular}
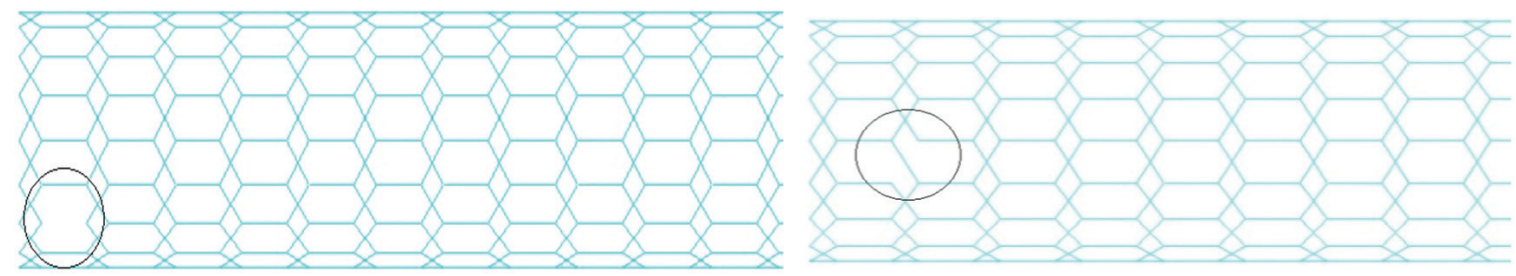

Figure 11. The position of a broken covalent bond in $(9,0)$ CNT leading to the highest reduction (right) and the lowest reduction (left) in CNT modulus.
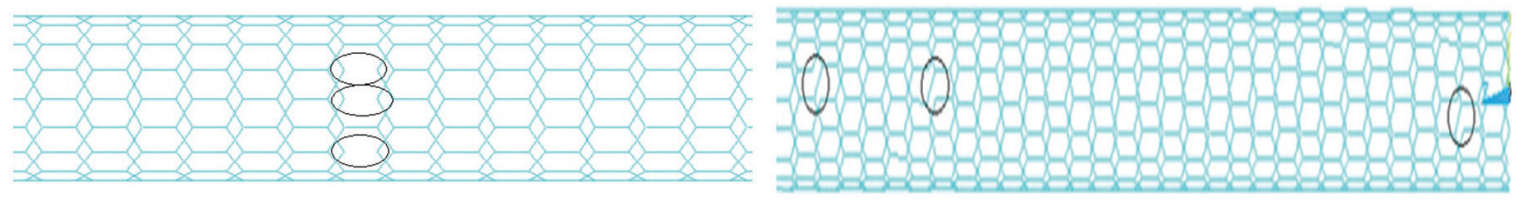

Figure 12. The locations of three broken covalent bonds in $(9,0)$ CNT leading to the highest reduction (left) and the lowest reduction (right) in CNT modulus. 

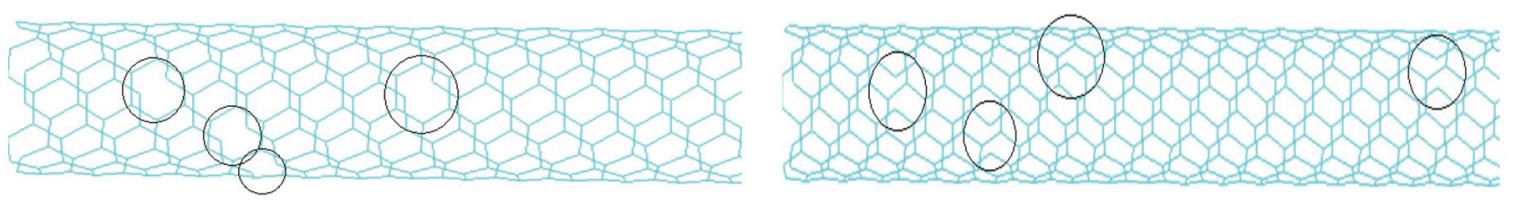

Figure 13. The locations of four broken covalent bonds in $(5,5) \mathrm{CNT}$ leading to the highest reduction (left) and the lower reduction (right) in CNT modulus.

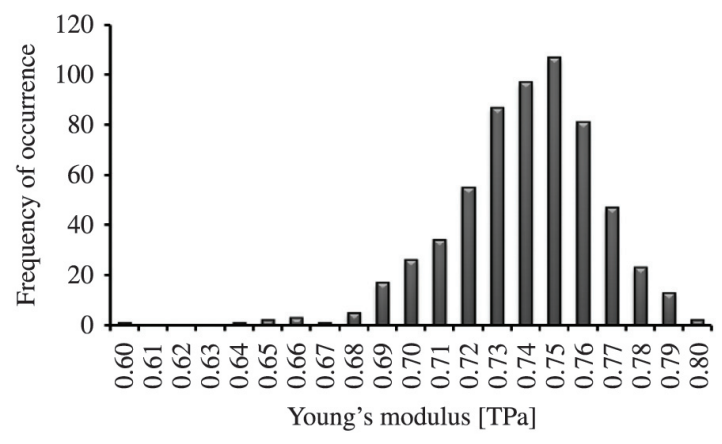

Figure 14. The frequency of defected CNT $(12,0)$ with $5 \%$ of the defect density in 600 runs.

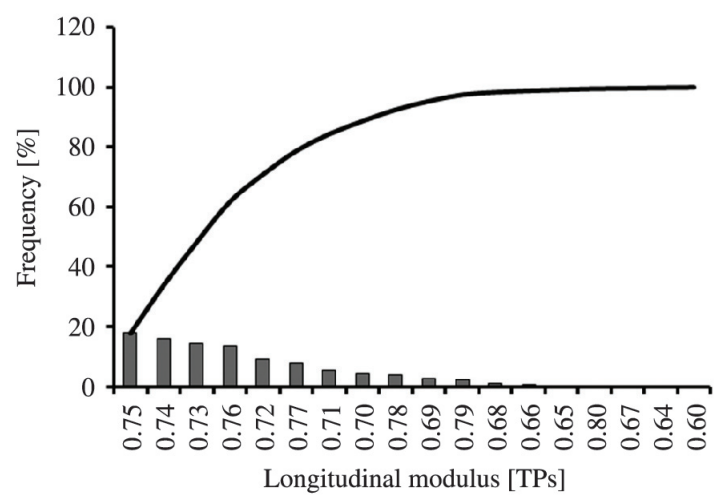

Figure 15. Pareto plot of defected CNT $(12,0)$ with $5 \%$ defect density.

value for the Young's modulus are not the same, Weibull distribution will be good candidate for a continuous probability density function. On the other hand, due to this fact that both mean and mode values are very close together, Gaussian distribution will be also examined. The general form of the Weibull function is presented in below equation $^{29}$ :

$$
W(E)=\left(\frac{k}{\lambda}\right)\left(\frac{E}{\lambda}\right)^{k-1} e^{-\left(\frac{E}{\lambda}\right)^{k}}
$$

where; $\mathrm{k}, \lambda$ and $\mathrm{E}$ are shape parameter, scale parameter and elastic modulus, respectively. For the presented results in Figure 14, shape and scale parameters are calculated 33.485 and 0.75 , respectively. The Gaussian and Weibull distributions for the Young's modulus of defected $(12,0)$ CNT with 5\% defect density are provided in Figure 16.

As shown in Figure 16, The Weibull distribution curve is slightly shifted to the right in comparison with Gaussian

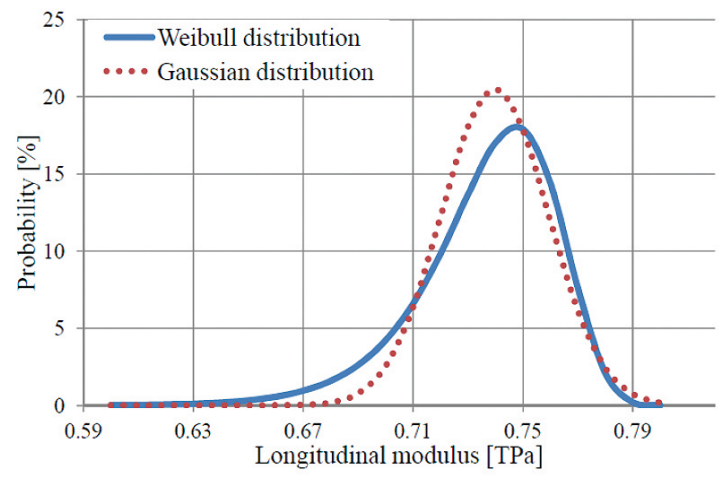

Figure 16. The Weibull and Gaussian plot of defected CNT $(12,0)$ with $5 \%$ of the defect density.

distribution curve. This behavior is originated from this fact that the accumulated probability of the Young's moduli greater than obtained mean value $(0.75 \mathrm{TPa})$ is $45 \%$; while the accumulated probability of the Young's moduli greater than mode value $(0.74 \mathrm{TPa})$ is $61 \%$.

\section{Conclusions}

The Young's modulus of defected CNT is obtained and compared with intact CNT to study the influence of defects on the mechanical property of the single walled CNT. Nanoscale continuum modeling using finite element modeling is employed for this purpose. The intrinsic defects induced during growth process of CNT called Stone-Wales defects and also vacancy defect appeared due to the chemical functionalization of the CNT are simulated and analyzed. Full stochastic modeling procedure is developed taking into account all numbers, orientations and locations of the defect as random parameters. Consequently, distribution of defects along the length and circumference of CNTs are considered as random parameters in order to be able to capture the real nature of defect topology. A computer code is written for this purpose to incorporate arbitrary number of defects into the discrete structure of CNT and reports the results.

The results demonstrate that the influence of S-W defects on the reduction of Young's modulus is negligible in comparison with than that of vacancy defects. Moreover, it is observed that the reduction rate of CNT Young's modulus will decrease by increasing number of S-W defects. The vacancy defect can reduce the Young's modulus of CNT about 12 to $13 \%$ when $2 \%$ of C-C bonds are broken in the CNT nanostructure. A detailed study on the topology of the vacancy defect is carried out and those configurations which will be led to the extreme values of Young's modulus are identified and discussed. Defect topology indicates that 
among all broken bonds, those bonds which are oriented more closely to the longitudinal axis of CNT have a greater influence on reduction of CNT Young's modulus. Moreover, local concentration of broken bonds will significantly reduce the Young's modulus in comparison with distributed broken bonds along the length of CNT. Finally, due to the stochastic modeling, a statistical analysis is performed to obtain probability of occurrence associated with different obtained results. It can be seen that influence of the vacancy defect which is usually arisen from functionalization of CNT is considerably more pronounced than that of S-W defects. Although, functionalization can improve the stress

\section{References}

1. Lau KT, Gu C and Hui D. A critical review on nanotube and nanotube/nanoclay related polymer composite materials. Composites Part B: Engineering. 2006; 37(6):425-436. http:// dx.doi.org/10.1016/j.compositesb.2006.02.020

2. Belin $\mathrm{T}$ and Epron F. Characterization methods of carbon nanotubes: a review. Materials Science and Engineering B. 2005; 119(2):105-118. http://dx.doi.org/10.1016/j. mseb.2005.02.046

3. Li F, Cheng HM, Bai S, Su G and Dresselhaus MS. Tensile strength of single walled carbon nanotubes directly measured from their macroscopic ropes. Applied Physics Letters. 2000; 77(20):3161-3163. http://dx.doi.org/10.1063/1.1324984

4. Li YJ, Wang KKL, Wei JQ, Gu ZY, Wang ZC, Luo JB et al. Tensile properties of long aligned double-walled carbon nanotube strands. Carbon. 2005; 43(1):31-35. http://dx.doi. org/10.1016/j.carbon.2004.08.017

5. Sinnott SB. Chemical functionalization of carbon nanotubes. Journal of Nanoscience and Nanotechnology. 2002; 2(2):113123. PMid:12908295. http://dx.doi.org/10.1166/jnn.2002.107

6. Bahr JL and Tour JM. Covalent chemistry of single-wall carbon nanotubes. Journal of Materials Chemistry. 2002; 12(7):19521958. http://dx.doi.org/10.1039/b201013p

7. Frankland SJV, Caglar A, Brenner DW and Griebel M. Molecular simulation of the influence of chemical cross-links on the shear strength of carbon nanotube polymer interfaces. Journal of Physical Chemistry: B. 2002; 106(12):3046-3048. http://dx.doi.org/10.1021/jp015591+

8. Buffa F, Abraham GA, Grady BP and Resasco D. Effect of nanotube functionalization on the properties of single-walled carbon nanotube/polyurethane composites. Journal of Polymer Science Part B: Polymer Physics. 2007; 45(4):490-501. http:// dx.doi.org/10.1002/polb.21069

9. Hao X, Qiang H and Xiaohu Y. Buckling of defective singlewalled and double-walled carbon nanotubes under axial compression by molecular dynamics simulation. Composites Science and Technology. 2008; 68(7-8):1809-1814. http:// dx.doi.org/10.1016/j.compscitech.2008.01.013

10. Yuan J and Liew K. Effects of vacancy defect reconstruction on the elastic properties of carbon nanotubes. Carbon. 2009; 47(6):1526-1533. http://dx.doi.org/10.1016/j. carbon.2009.01.048

11. Ghavamian A, Rahmandoust M and Öchsner A. A numerical evaluation of the influence of defects on the elastic modulus of single and multi-walled carbon nanotubes. Computational transferring phenomenon from surrounding polymer to the embedded CNT, it can significantly reduce the Young's modulus of CNT. Therefore, this should be taken into account when functionalized CNT is incorporated into polymer.

\section{Acknowledgment}

The authors acknowledge the financial support provided by the Iranian National Science Foundation (INSF) under contract 91001046 .

Materials Science. 2012; 62:110-116. http://dx.doi. org/10.1016/j.commatsci.2012.05.003

12. Zhao J, Wu J, Jiang JW, Lu L, Zhang Z and Rabczuk T. Thermal conductivity of carbon nanocoils. Applied Physics Letters. 2013; 103(23):233511. http://dx.doi.org/10.1063/1.4839396

13. Shokrieh MM and Rafiee R. Stochastic multi-scale modeling of CNT/polymer composites. Computational Materials Science. 2010; 50(2):437-446. http://dx.doi.org/10.1016/j. commatsci.2010.08.036

14. Spanos PD and Kontsos A. A multiscale Monte Carlo finite element method for determining mechanical properties of polymer nanocomposites. Probabilistic Engineering Mechanics. 2008; 23(4):456-470. http://dx.doi.org/10.1016/j. probengmech.2007.09.002

15. Wu Y, Zhang X, Leung AYT and Zhong W. An energyequivalent model on studying the mechanical properties of single-walled carbon nanotubes. Thin-Walled Structures. 2006; 44(6):667-676. http://dx.doi.org/10.1016/j.tws.2006.05.003

16. Grady BP. Carbon nanotube composites. University of Oklahoma. Available from: <http://coecs.ou.edu/Brian.P.Grady/ nanotube.html>.

17. Qi X, Guo X and Zheng C. Density functional study the interaction of oxygen molecule with defect sites of graphene. Applied Surface Science. 2012; 259:195-200. http://dx.doi. org/10.1016/j.apsusc.2012.07.018

18. Tserpes K and Papanikos P. Finite element modeling of single-walled carbon nanotubes. Composites Part B: Engineering. 2005; 36(5):468-477. http://dx.doi.org/10.1016/j. compositesb.2004.10.003

19. Li C and Chou T. A structural mechanics approach for the analysis of carbon nanotubes. International Journal of Solids and Structures. 2003; 40(10):2487-2499. http://dx.doi. org/10.1016/S0020-7683(03)00056-8

20. Koloczek J, Young-Kyun K and Burian A. Characterization of spatial correlations in carbon nanotubes-modelling studies. Journal of Alloys and Compounds. 2001; 328(2):222-225. http://dx.doi.org/10.1016/S0925-8388(01)01298-1

21. Ansys Inc. Theory manual. SAS IP Inc.; 2003.

22. Shokrieh MM and Rafiee R. Prediction of Young's modulus of graphene sheets and carbon nanotubes using nanoscale continuum mechanics approach. Materials and Design. 2010; 31(2):790-795. http://dx.doi.org/10.1016/j.matdes.2009.07.058

23. Kleiber M and Hien TD. The stochastic finite element method. New York: John Wiley \& Sons; 1992. 
24. Saxena KK and Lal A. Comparative molecular dynamics simulation study of mechanical properties of carbon nanotubes with number of stone-wales and vacancy defects. Procedia Engineering. 2012; 38:2347-2355, 2012. http://dx.doi. org/10.1016/j.proeng.2012.06.280

25. Sharma K, Saxena K and Shukla M. Effect of multiple Stone-Wales and vacancy defects on the mechanical behavior of carbon nanotubes using molecular dynamics. Procedia Engineering. 2012; 38:3373-3380. http://dx.doi.org/10.1016/j. proeng.2012.06.390

26. Chen L, Zhao Q, Gong Z and Zhang $H$. The effects of different defects on the elastic constants of single-walled carbon nanotubes. In: Proceedings of the 5th IEEE International Conference on Nano/Micro Engineered and Molecular Systems; 2010, Xiamen, China.

27. Xiao JR and Gillespie JW. Fracture behaviors of graphene sheets and carbon nanotubes. In: Mikhailov S, editor. Physics and applications of graphene: theory. InTech; 2011.

28. Shofner ML, Khabashesku VN and Barrera EV. Processing and mechanical properties of fluorinated single-wall carbon nanotube-polyethylene composites. Chemistry of Materials. 2006; 18(4):906-913. http://dx.doi.org/10.1021/cm051475y

29. Kreyszig E. Advanced engineering mathematics. 4th ed. John Wiley \& Sons; 1979. 